\title{
Diplopia and ocular pain due to orbital myositis
}

\section{Rosa I. Arvizu-Rivera ${ }^{1}$ (D) - Rita A. Pineda-Sic ${ }^{2}$ (D) - Jesus Alberto Cardenas-de la Garza ${ }^{2}$ (D) - Dionicio A. Galarza-Delgado ${ }^{2}$ (D) Miguel A. Villarreal-Alarcon ${ }^{2}$ (10}

Received: 11 March 2020 / Revised: 30 April 2020 / Accepted: 1 May 2020 / Published online: 23 June 2020

(C) International League of Associations for Rheumatology (ILAR) 2020

\section{Presentation}

A 19-year-old man presented to the emergency department because of sudden onset of diplopia and ocular pain. He reported a 1-week history of malaise and headache. He denied travels or rash. Physical examination revealed ptosis and limited and painful abduction of the right eye. Pupillary light reflexes and dilated fundus examination were normal. Complete blood count, biochemical profile, erythrocyte sedimentation rate, $\mathrm{C}$-reactive protein, and thyroid function tests were within normal limits. Antinuclear antibodies, antineutrophil cytoplasmic antibodies (ANCA), antimyeloperoxidase, and anti-proteinase 3 were negative. Fat suppression T1-weighted magnetic resonance imaging (MRI) showed right lateral rectus muscle enlargement with contrast enhancement, and tendinopathy (Fig. 1).

A trial of prednisone $1 \mathrm{mg} / \mathrm{kg}$ daily was initiated with symptom resolution after 2 days. Based on clinical characteristics, MRI findings, and treatment response, diagnosis of orbital myositis (OM) was reached. Methotrexate $15 \mathrm{mg}$ weekly was started, and oral steroids were slowly tapered without recurrence after 4 months of follow-up.

\section{Discussion}

OM is part of the spectrum of idiopathic orbital inflammation syndromes, which constitute a group of inflammatory disorders affecting the orbit and lacking systemic or local secondary causes. OM is characterized by acute/subacute unilateral

Miguel A. Villarreal-Alarcon

mavillar@yahoo.com

1 Department of Internal Medicine, Hospital Universitario "Dr. Jose Eleuterio Gonzalez", Monterrey, Nuevo Leon, Mexico

2 Department of Rheumatology, Hospital Universitario "Dr. Jose Eleuterio Gonzalez", Monterrey, Nuevo Leon, Mexico painful ophthalmoplegia, diplopia, ptosis, and conjunctival injection. The muscle most often involved is the lateral rectus [1].

Differential diagnosis includes Grave's orbitopathy (GO), lymphoma, infectious diseases, myasthenia gravis, ANCAassociated vasculitis, and other autoimmune diseases. MRI can help distinguish OM from GO. In OM, MRI shows thickening and contrast enhancement of the muscle belly with myotendinous junction involvement. In contrast, $\mathrm{GO}$ is usually bilateral, affects more commonly the inferior rectus muscle, and spares the myotendinous junction $[2,3]$.

Diagnosis of OM is reached after exclusion of other disorders, in combination with clinical, imaging, and laboratory findings and a rapid response to corticosteroids [2, 3]. Immunosuppressants are useful for disease control and relapse prevention of OM $[2,3]$.

Code availability We did not use any new software application or custom code.

Data availability Additional information is available from the corresponding author on reasonable request.

\section{Compliance with ethical standards}

Disclosures None.

Ethics approval Ethical approval was not required because of the anonymity of the images.

Consent to participate Patient's consent to participate and for publication was obtained.

Consent for publication Patient's consent to participate and for publication was obtained.

\section{References}

1. Montagnese F, Wenninger S, Schoser B (2016) "Orbiting around" the orbital myositis: clinical features, differential diagnosis and 
Fig. 1 Fat suppression T1weighted images (a axial view, b coronal view) showing fusiform thickening and contrast enhancement of the right lateral rectus (white arrow) with tendon involvement
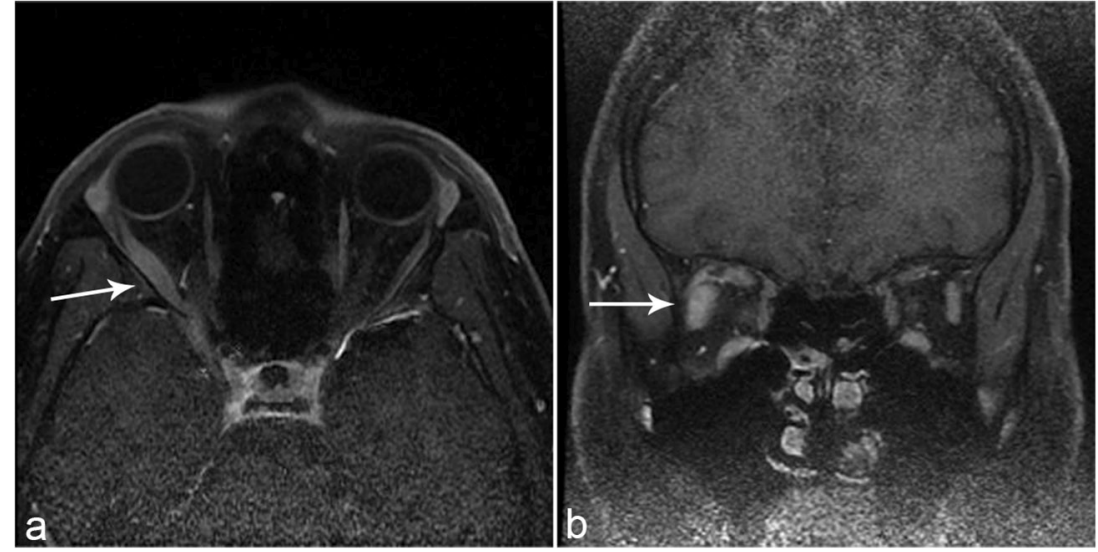

therapy. J Neurol 263:631-640. https://doi.org/10.1007/s00415-0157926-x

2. Avni-Zauberman N, Tripathy D, Rosen N, Ben Simon GJ (2012) Relapsing migratory idiopathic orbital inflammation: six new cases and review of the literature. Br J Ophthalmol 96:276-280. https://doi. org/10.1136/bjo.2010.191866
3. Yuen SJA, Rubin PAD (2003) Idiopathic orbital inflammation: distribution, clinical features, and treatment outcome. Arch Ophthalmol 121:491-499. https://doi.org/10.1001/archopht.121.4.491

Publisher's note Springer Nature remains neutral with regard to jurisdictional claims in published maps and institutional affiliations. 